\title{
A Necessary and Sufficient Condition for Optimal Transmission Power in Wireless Packet Networks with ARQ Capability
}

\author{
Hanbyul Seo and Byeong Gi Lee \\ School of Electrical Engineering and INMC, Seoul National University
}

\begin{abstract}
In this paper, we investigate the energy minimization problem for wireless packet networks with automatic repeat request (ARQ) capability. We formulate the problem under constrained packet delay and reliability, and derive a necessary and sufficient condition for optimal transmission power that achieves optimality regardless of the delay and reliability constraints. Numerical examples reveal that a significant amount of energy is saved by adopting the optimal transmission power and the performance gain is strongly correlated with the decreasing property of the frame error rate.
\end{abstract}

\section{INTRODUCTION}

Recently, energy efficiency has emerged as one of the major research issues in wireless networks [1-3]. As most wireless devices such as lap-top computers, cellular phones and wireless sensor nodes operate with limited battery power, it is very important to find ways of maximizing the device lifetime by efficiently utilizing the limited energy. In addition, an efficient energy management scheme can mitigate the channel interference to other devices, enabling a harmonized channel sharing among multiple wireless devices.

On the other hand, a growing demand for wireless data access motivates researches on energy efficient operations for packet-switched data services. Data services, in general, have some distinctive features over conventional voice services, whose typical example is the requirement of high reliability. For a reliable operation of the upper-layer protocols like TCP in wireless networks, it is necessary to keep the frame loss rate at low level (e.g. $10^{-3}$ or $10^{-4}$ ) $[4,5]$. Another distinctive feature of data services is the delay tolerance, which enables the deployment of an automatic repeat request (ARQ) protocol in wireless data networks. By retransmitting the erred frames repeatedly, an ARQ protocol can help to improve the performance of the upper-layer protocols significantly at the expense of an increased delay [6].

The above features of wireless data services have triggered extensive researches for exploiting energy efficient operations of wireless packet data networks [3, 5, 7-9]: Bambos and Kandukuri [3] proposed a new power control scheme that can minimize a cost function composed of buffer size and transmission power but it did not support the packet-wise delay constraint and rendered only semi-analytical solution. Song

This research was supported by University IT Research Center Project. et. al. [5] proposed a signal-to-interference ratio (SIR) and rate control scheme but it relied on implicit and complex iterative calculations and supported only local optimality. Rajan et. al. [7] and Wang et. al. [8] proposed optimal transmission schemes under constrained buffer states but did not consider ARQ protocols, assuming error-free transmissions. Liu et. al. [9] considered adaptive transmissions with ARQ protocols but did not consider how to minimize the energy consumption.

In this paper, we are going to present an optimal transmission power control scheme that minimizes the average transmission energy consumption for ARQ protocols. In formulating the energy minimization problem, we consider two kinds of constraints: One is the delay constraint that limits the number of retransmissions of a frame and the other is the reliability constraint that limits the residual loss rate after applying ARQ procedures. Based on the formulation, we derive a necessary and sufficient condition that achieves the optimality. As will become clear throughout the relevant reasonings in Section III, the optimal transmission power at an ARQ stage is determined by a global rule, regardless of the delay and reliability constraints. Based on this optimality condition, we also derive some properties of the optimal transmission power. As will be demonstrated through numerical examples in Section IV, a significantly large amount of energy can be saved by adopting the above optimal transmission power and the performance gain of the optimal transmission power turns out to be strongly correlated with the decreasing property of the frame error rate.

\section{System Model And Problem Formulation}

We consider the single-hop packet transmission over a wireless channel equipped with a radio link protocol (RLP). We assume that the transmitter has no information about the instantaneous channel condition and the channel state (including the interference from other devices) varies independently at each transmission. In this case, we can characterize the wireless channel in terms of frame error rate (FER) such that for a given transmission power $P(\geq 0)$ the corresponding FER is determined by $f(P) .{ }^{1}$ Obviously, the FER function $f(P)$

\footnotetext{
${ }^{1}$ The FER $f(P)$ means the frame error rate averaged with respect to the channel state. In other words, $f(P) \equiv \mathrm{E}_{\alpha}\left[f_{\alpha}(P)\right]$ where $f_{\alpha}(P)$ denotes the frame error rate with the channel state $\alpha$.
} 


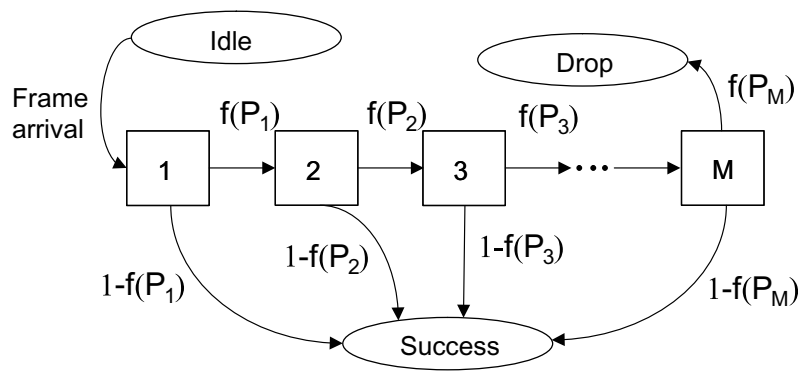

Fig. 1. State transition diagram of the ARQ procedure.

is a monotonically decreasing function with $f(0)=1$ and $f(P)>0$. In addition, we assume that $f(P)$ is analytic and strictly convex, which is consistent with the assumptions in the references $[5,10]$. This means that $f^{\prime}(P)<0$ and $f^{\prime \prime}(P)>0$ for all $P \geq 0$.

In order to overcome the unreliable nature of wireless channels, we adopt a simple stop-and-wait ARQ protocol within the RLP. Fig. 1 depicts the state transition diagram of the ARQ procedure: Initially, the ARQ is in the idle state and the transmitter waits for a frame arrival. Once a frame arrives, the transmitter transmits it to the receiver with a transmission power $P_{1}$. This transmission errs with probability $f\left(P_{1}\right)$ and succeeds with probability $1-f\left(P_{1}\right)$. If the transmission succeeds, the receiver sends an ACK message to the transmitter and the overall ARQ procedure finishes successfully. Otherwise, the receiver sends a NAK message and the transmitter retransmits the erred frame with a transmission power $P_{2}$. The ARQ procedure repeats transmission up to $M$ times with the transmission power varying according to the vector $\mathbf{P}=\left[P_{1}, \cdots, P_{M}\right]\left(\in \mathcal{R}_{+}^{M}\right)$ until the frame is received correctly. We call the maximum transmission number $M$ the delay constraint, which is determined such that the requirement on the maximum tolerable delay of a frame is met. If the $M$-th transmission of a frame fails, the frame is dropped and the RLP begins the ARQ procedure for the next frame. We call this residual error rate caused by frame drop the frame drop rate (FDR). We say the system is at stage $m$ if the current transmission corresponds to the $m$-th transmission of a frame.

We set the system objective to the minimization of the average transmission energy, $\bar{P}$, that is required to deliver a frame under a given reliability constraint which imposes a reliability requirement value $\epsilon$ on the FDR. Then the energy minimization problem is formulated in the mathematical form (we call it Problem SH)

$$
\begin{aligned}
& \text { minimize } \bar{P} \equiv \sum_{m=1}^{M} P_{m} \prod_{i=1}^{m-1} f\left(P_{i}\right) \\
& \text { subject to } F D R \equiv \prod_{m=1}^{M} f\left(P_{m}\right) \leq \epsilon .
\end{aligned}
$$

We denote by $\mathbf{P}^{*}$ the optimal transmission power vector, that is, the solution of the Problem SH.

\section{Optimal TRANSMission POWER}

\section{A. Derivation of the Optimal Transmission Power}

In general, the Problem SH in the previous section is not a convex optimization problem in spite of the convexity of $f(P)$. The objective $\bar{P}$ may not be a convex function of $\mathbf{P}$ as the Hessian of $\bar{P}$ is not necessarily positive semidefinite. This property disables applying the Lagrange multiplier method, so we solve the problem in an alternative way.

First, we can easily derive the following property that the optimal transmission power vector holds.

Proposition 1: For the solution of the Problem SH, $\mathbf{P}^{*}$, it holds that

$$
F D R=\prod_{m=1}^{M} f\left(P_{m}^{*}\right)=\epsilon .^{2}
$$

This proposition implies that the optimal transmission power meets the FDR constraint with equality. In addition, we can establish the following proposition. ${ }^{3}$

Proposition 2: If $\mathbf{P}^{*}$ is the solution of the Problem $\mathrm{SH}$, then $P_{m}^{*}>0$ for $m=1,2, \cdots, M$.

Summary of proof: We prove the proposition by contradiction. First, suppose that a vector $\mathbf{Q}=\left[Q_{1}, Q_{2}, \cdots, Q_{M}\right]$ having a zero element $Q_{m}=0$ for an $m \neq M$ is the solution of the Problem SH. We consider another vector $\mathbf{P}=\left[Q_{1}, \cdots, Q_{m-1}, Q_{m+1}, \cdots, Q_{M-1}, P, P\right]$ for a $P(>0)$ that meets $Q_{M}=P+P f(P)$. Then, it consumes the same amount of energy as $\mathbf{Q}$ does. Based on this, we can prove that $F D R(\mathbf{P})<F D R(\mathbf{Q})$. When $Q_{M}=0$, the vector $\tilde{\mathbf{Q}}=\left[Q_{1}, \cdots, Q_{M-2}, Q_{M}, Q_{M-1}\right]$ yields the same FDR and the same energy consumption as $\mathbf{Q}$ does, so we can prove that $F D R(\mathbf{P})<F D R(\mathbf{Q})$ in this case too. This means that for the given optimal vector $\mathbf{Q}$ that contains a zero element, there exists another power vector $\mathbf{P}$ that consumes the same energy but yields a smaller FDR, which is a contradiction.

This proposition implies that the transmission power at each stage should be set to a positive level. It also indicates that the optimal transmission power vector is not located at the boundary of the candidate set $\left\{\mathbf{P} \mid F D R=\epsilon, \mathbf{P} \in \mathcal{R}_{+}^{M}\right\}$.

We now derive the necessary and sufficient condition for the optimal transmission power. By Proposition 2, it is established that the optimal vector is not a boundary vector of the candidate set. Therefore, if a transmission power vector is optimal, the gradient of the objective function $\bar{P}$ becomes zero for the vector, while satisfying the FDR constraint with equality. The following proposition presents this necessary condition of the optimal transmission power vector in terms of $P_{m}^{*}$ and $P_{m+1}^{*}$.

\footnotetext{
${ }^{2}$ We omit the proof as it is trivial.

${ }^{3}$ Refer to [11] for the detailed proofs of all the propositions in this paper.
} 
Proposition 3: For the optimal transmission power vector $\mathbf{P}^{*}$, it holds that

$$
\frac{f\left(P_{m+1}^{*}\right)}{f^{\prime}\left(P_{m+1}^{*}\right)}-P_{m+1}^{*}-\frac{1}{f^{\prime}\left(P_{m}^{*}\right)}=0
$$

for $m=1,2 \cdots M-1$.

Summary of proof: We can prove the proposition by the mathematical induction. For the case of $M=2, P_{2}$ is a function of $P_{1}$ for a fixed FDR constraint $\epsilon$ by Proposition 1. So, by expressing $P_{2}$ in terms of $P_{1}$ and differentiating $\bar{P}$ with respect to $P_{1}$, we can derive the relation in (3) for $M=2$. Then, we note the recursive nature of the Problem $\mathrm{SH}$, which means that, once $P_{1}$ is fixed to an arbitrary value, the remaining problem (i.e., determining $P_{2}, P_{3}, \cdots, P_{M}$ ) becomes another Problem SH with the reduced transmission number $M-1$. If we apply this property to the mathematical induction, we can prove that the relation in (3) holds for all $M>2$.

This proposition helps to establish the uniqueness of the optimal transmission power vector $\mathbf{P}^{*}$ as follows:

Proposition 4: For a feasible FDR constraint $\epsilon$, the transmission power vector that satisfies the relation in (3) is unique.

Summary of proof: First, due to the strictly convex property of $f(P)$, we can prove that, for a given $P_{m}^{*}$, there exists only one $P_{m+1}^{*}$ that satisfies the relation in (3). This establishes that once $P_{1}$ is determined for the given FDR $\epsilon$, then $P_{2}, P_{3}, \cdots, P_{M}$ are uniquely determined by the relation (3). Now suppose that for the given FDR value $\epsilon$ there exist two different power vectors $\mathbf{P}^{*}$ and $\mathbf{Q}^{*}$, with $P_{1}^{*}>Q_{1}^{*}$, that satisfy (3). Then we can show that $P_{m+1}^{*}>Q_{m+1}^{*}$, $m=1,2, \cdots, M-1$. If we feed back this relation to (2), we get $\epsilon=\prod_{m=1}^{M} f\left(P_{m}^{*}\right)<\prod_{m=1}^{M} f\left(Q_{m}^{*}\right)=\epsilon$, which is a contradiction.

Proposition 4 reveals that the relation in (3) is not only the necessary condition but also the sufficient condition of the optimality. Therefore, by combining Propositions 3 and 4, we can establish the following theorem:

Theorem 1: A transmission power vector $\mathbf{P}$ is the optimal solution of Problem SH if and only if the relation in (3) holds for $m=1,2, \cdots M-1$.

\section{B. Properties of the Optimal Transmission Power}

Theorem 1 is a powerful theorem that dictates some interesting properties of the optimal transmission power vector.

First, it is noteworthy that the optimal transmission power at the $(m+1)$-th stage, $P_{m+1}^{*}$, is determined only by the power at the $m$-th stage, $P_{m}^{*}$, regardless of the packet delay and reliability constraints. We may refer this as the memoryless property of the optimal transmission power vector. Due to this memoryless property, it is utmost important, when determining the optimal transmission power vector, to determine the initial transmission power $P_{1}^{*}$ precisely with the FDR constraint met with equality.

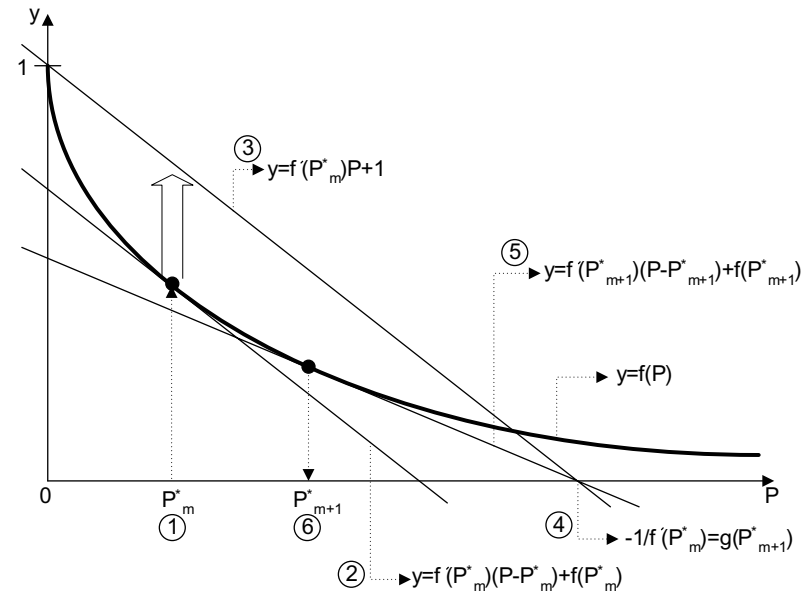

Fig. 2. Graphical representation of determining $P_{m+1}^{*}$ from $P_{m}^{*}$ by utilizing the FER function $y=f(P)$.

Secondly, the optimal transmission power vector meets an increasing property as follows:

Proposition 5: For an optimal transmission power vector $\mathbf{P}^{*}$, it holds that $P_{1}^{*} \leq P_{2}^{*} \leq \cdots \leq P_{M}^{*}$.

Summary of proof: We can prove the proposition by showing that the assumption $P_{m}^{*}>P_{m+1}^{*}$ for an $m=1,2, \cdots$, or $M-1$ leads to contradiction.

This proposition implies that, for an optimal energy consumption, a failed frame should be retransmitted with an increased transmission power.

Third, once the optimal transmission power vector is determined for a channel gain, it is possible to maintain the optimality for a changed channel gain by simply modifying the power vector in compensation for the change. We may put this in a proposition as follows:

Proposition 6: Let a transmission power vector $\mathbf{P}^{*}$ be the solution of the Problem SH with the FDR constraint $\epsilon$ for the frame error rate $f(P)$. Then, $\mathbf{Q}^{*}=\alpha \mathbf{P}^{*}(\alpha>0)$ becomes the solution of the same problem for the frame error rate $\hat{f}(P)=$ $f(P / \alpha)$.

Summary of proof: Since $\hat{f}^{\prime}\left(Q_{m}^{*}\right)=f^{\prime}\left(P_{m}^{*}\right) / \alpha$, we can show that the relation in (3) holds for the power vector $\mathbf{Q}^{*}$ and FER $\hat{f}(P)$, which proves the proposition by Theorem 1 .

For example, we suppose that the optimal transmission power vector was given by $[0 \mathrm{~dB}, 2 \mathrm{~dB}, 5 \mathrm{~dB}]$ in the beginning and the receiver moves closer to the transmitter to give the effect of increasing the channel gain by $3 \mathrm{~dB}$. Then the new optimal transmission power vector becomes $[-3 \mathrm{~dB},-1 \mathrm{~dB}, 2$ $\mathrm{dB}]$, which is obtained simply by decreasing each transmission power by $3 \mathrm{~dB}$. This property helps us to avoid repeated computations of the optimal transmission power vector with respect to the change of the channel gain.

Fourth, once the optimal initial transmission power $P_{1}^{*}$ is determined, it is possible to determine $P_{2}^{*}, P_{3}^{*}, \cdots, P_{M}^{*}$ 


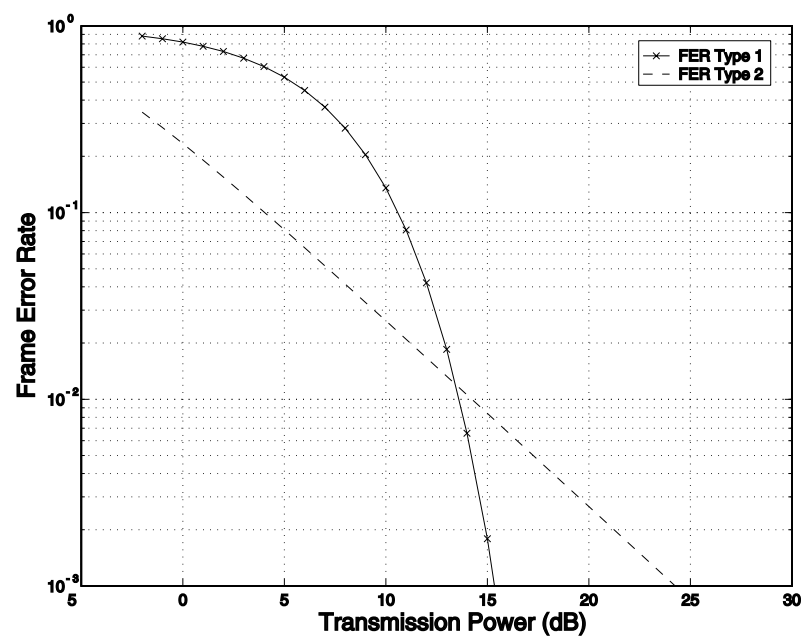

Fig. 3. Two different types of frame error rate function.

graphically as illustrated in Fig. 2. In the figure, the solid curve represents the FER curve $f(P)$ which is monotonically decreasing and strictly convex (see Section II). Given an optimal transmission power $P_{m}^{*}$, we first determine the tangential slope of the FER curve at $P=P_{m}^{*}, f^{\prime}\left(P_{m}^{*}\right)$. Then the value $-1 / f^{\prime}\left(P_{m}^{*}\right)$ in (3) corresponds to the cross-point of the line $y=f^{\prime}\left(P_{m}^{*}\right) P+1$ and the horizontal axis. On the other hand, the term $P_{m+1}^{*}-f\left(P_{m+1}^{*}\right) / f^{\prime}\left(P_{m+1}^{*}\right)$ in (3) corresponds to the cross-point of the line $y=f^{\prime}\left(P_{m+1}^{*}\right)\left(P-P_{m+1}^{*}\right)+f\left(P_{m+1}^{*}\right)$, which is tangential to $f(P)$ at $\left(P_{m+1}^{*}, f\left(P_{m+1}^{*}\right)\right)$, and the horizontal axis. The equality (3) means that the two crosspoints coincide. Therefore, we can determine the optimal transmission power $P_{m+1}^{*}$ by determining the tangential point at which the above line (which passes through the crosspoint) hits the curve $f(P)$. By repeating this process, we can determine all the optimal transmission powers graphically.

\section{NumericAl RESULts}

In order to examine the energy efficiency of the optimal transmission power vector through some numerical examples, we consider two different types of the FER function as shown in Fig. 3: The type 1 is a simple exponential function given by

$$
f(P)=e^{-\beta P},
$$

which was used as an approximated error probability in [3, 12]. We set $\beta=0.2$ to make the curve comparable with the other type in the FER range of practical interest. The type 2 is the FER function that models the error rate in fading channel by

$$
f(P)=1-e^{-1 / F(P)}
$$

where $F(P)$ represents the fade margin. We set $F(P)=$ $3.75 P$ as in the literature $[5,13]{ }^{4}$

\footnotetext{
${ }^{4}$ Note that the type 2 FER is not convex for all $P \geq 0$ but is convex for $f(P)<1-e^{-2} \approx 0.8647$, which indicates that the type 2 FER may be assumed convex in the region of interest.
}

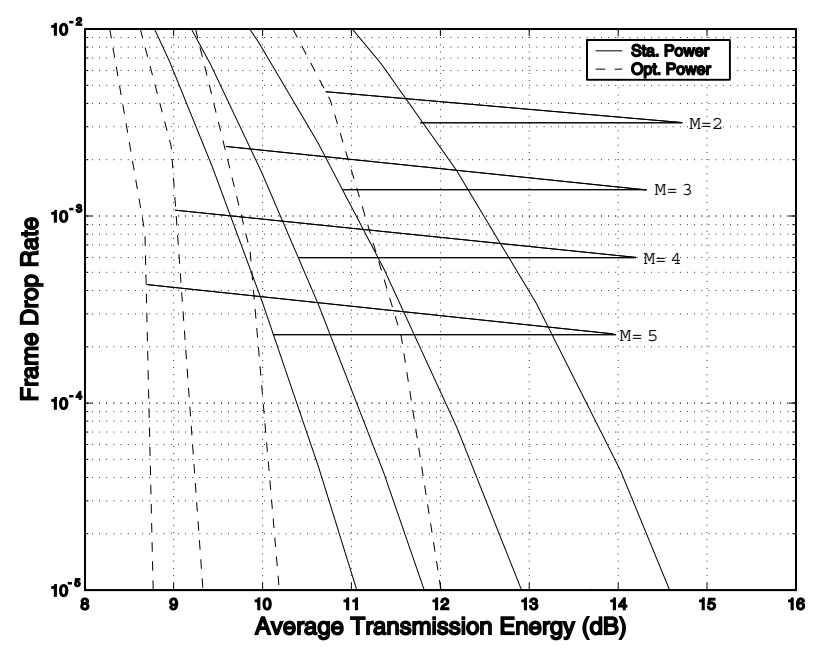

(a)

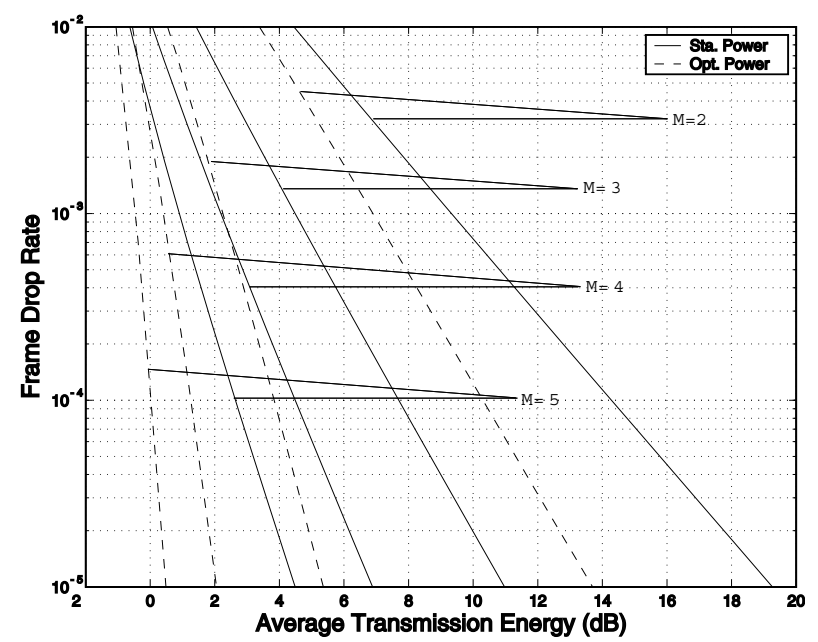

(b)

Fig. 4. Relation between the frame drop rate and the average transmission energy for (a) FER type 1, (b) FER type 2.

Fig. 4 depicts the relation between the frame drop rate and the average transmission energy for the two different FER types. For performance comparison, we overlaid the curves for the stationary power vector case (i.e., $P_{1}=P_{2}=\cdots=P_{M}$ ) over the optimal power case. We observe that the optimal power vector outperforms the stationary power vector in that the average transmission energy required to achieve a given level of FDR is much smaller for the optimal vector case. We also observe that the FDR decreases more rapidly for a larger number of $M$ than for a smaller number. In this context, we may interpret the transmission number $M$ as a kind of diversity order that represents the number of independent delivery paths connecting the transmitter and the receiver.

Fig. 5 plots the amount of energy saved by using the optimal power vector instead of the stationary power vector. We observe that the performance gain of the optimal power increases as the FDR constraint $\epsilon$ becomes strict. In addition, we observe that the performance gain decreases as 


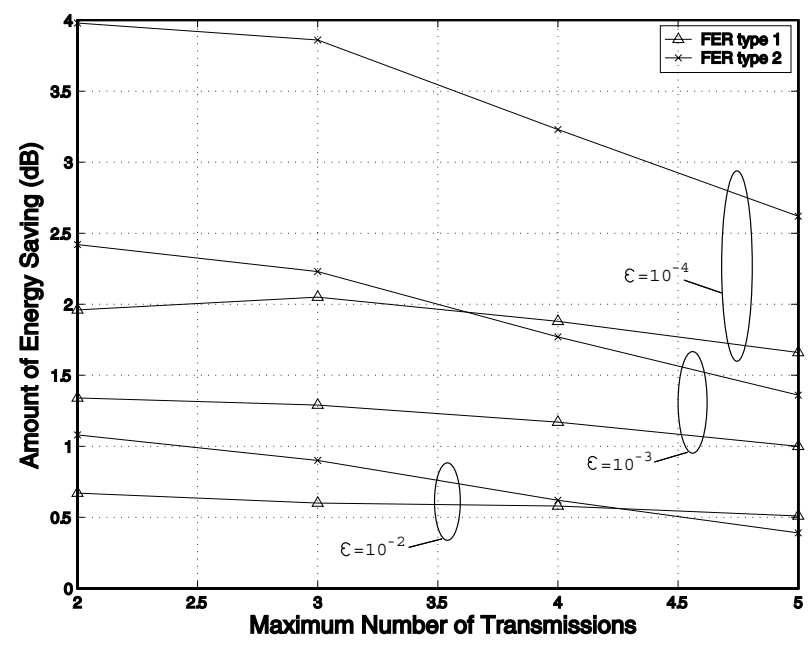

Fig. 5. The amount of energy saving gained by using the optimal transmission power vector instead of the stationary power vector.

the maximum number of transmissions $M$ increases. When $\epsilon=10^{-2}$, the performance gain is similar for the two different FER types with the difference limited within $0.5 \mathrm{~dB}$. However, when $\epsilon=10^{-4}$, the performance gain of the type 2 case is much larger than that of the type 1 case. This may be interpreted in terms of the FER curves in Fig. 3: We find that the performance gain of the optimal power over the stationary power increases as the slope of the FER curve gets gentler. This is because, if the FER curve decreases more slowly, the cross-point $-1 / f^{\prime}\left(P_{m}^{*}\right)$ in Fig. 2 moves to the right-side, resulting in a larger gap between $P_{m}^{*}$ and $P_{m+1}^{*}$. If we compare the decreasing property of the two FER types, we find that the slope of the FER type 1 is similar to the type 2 in the range $F E R>10^{-1}$, but becomes steeper beyond that range. So when the FER of each transmission lies in the range that yield the FDR of $10^{-4}$, the performance gain of the type 2 becomes much larger than that of the type 1 .

\section{COnClusions}

In this paper, we have investigated how to minimize the average energy consumption while guaranteeing a required level of reliability in the wireless packet networks with ARQ capability. We formulated the energy minimization problem as a constrained optimization problem, then derived the necessary and sufficient condition of the optimal transmission power at each ARQ stage. It turned out that the optimality rule has the memoryless property, that is, the optimal transmission power at a stage is determined only by the power at the previous stage. Besides, we have investigated some interesting properties of the optimal power vector such as the increasing property of optimal transmission power and the maintenance of the optimality in compensation for the change of channel gain. Numerical results that we performed for two different FER functions revealed that a significant amount of energy can be saved by determining the transmission power optimally.
The theorem and propositions established in this paper are noteworthy in that they establish for the first time the explicit expressions of the optimal transmission power that minimizes the average energy consumption in the wireless packet networks with ARQ capability. The theorem of the optimal transmission power that we have derived in this paper are applicable to any cases with arbitrary delay and reliability constraints, so it can be effectively applied to wireless ARQ protocols in various different communication environments. In addition, the propositions enable us to explain various properties, including the relations between the decreasing property of the FER curve and the optimal power vector. In particular, the propositions have established that, in the environment where an additional increment of transmission power does not contribute much in achieving the required reliability, it is more advantageous to utilize the additional power at the next ARQ stage. This finding enables an effective design of system parameters such as the maximum number of retransmissions according to the communication environments, which are characterized in terms of the FER curve.

\section{REFERENCES}

[1] I. F. Akyildiz, W. Su, Y. Sankarasubramaniam, and E. Cayirci, "A survey on sensor networks," IEEE Commun. Mag., vol. 40, pp. 102-114, Aug. 2002.

[2] V. Raghunathan, C. Schurgers, S. Park, and M. B. Srivastava, "Energy aware wireless microsensor networks," IEEE Sig. Proc. Mag., vol. 19, pp. 40-50, Mar. 2002.

[3] N. Bambos and S. Kandukuri, "Power-controlled multiple access schemes for next-generation wireless packet networks," IEEE Wireless Commun., vol. 9, pp. 58-64, June 2002.

[4] G. Xylomenos, G. C. Polyzos, P. Mähönen, and M. Saaranen, "TCP performance issues over wireless links," IEEE Commun. Mag., vol. 39, pp. 52-58, Apr. 2001.

[5] L. Song and N. B. Mandayam, "Hierarchical SIR and rate control on the forward link for CDMA data users under delay and error constraints," IEEE J. Select. Areas Commun., vol. 19, pp. 1871-1881, Oct. 2001.

[6] K. Premkumar and A. Chockalingam, "Performance analysis of RLC/MAC and LLC layers in a GPRS protocol stack," IEEE Trans. Veh. Technol. vol. 53, pp. 1531-1546, Sept. 2004.

[7] D. Rajan, A. Sabharwal, B. Aazhang, "Delay-bounded packet scheduling of bursty traffic over wireless channels," IEEE Trans. Info. Theory, vol. 50, pp. 125-144, Jan. 2004.

[8] H. Wang, N. B. Mandayam, "A simple packet transmission scheme for wireless data over fading channels," IEEE Trans. Commun. vol. 52, pp. 1055-1059, July 2004.

[9] Q. Liu, S. Zhou, and G. B. Giannakis, "Cross-Layer combining of adaptive modulation and coding with truncated ARQ over wireless links," IEEE Trans. Wireless Commun., vol. 3, pp. 1746-1754, Sept. 2004.

[10] S. Kumar, S. Nanda, and A. Falconi, "Reverse link power control performance for high speed data over CDMA," TIA Contribution, TR45.5.4/97.11, 1997.

[11] H. Seo and B. G. Lee, "Optimal transmission power for singleand multi-hop links in wireless packet networks with ARQ capability," submitted to IEEE Trans. Commun., Sept. 2005. Available at http://tsp.snu.ac.kr/ shb.

[12] A. J. Goldsmith and S.-G. Chua, "Variable-rate variable-power MQAM for fading channels," IEEE Trans. Commun., vol. 45, pp. 1218-1230, Oct. 1997.

[13] M. Zorzi, R. Rao, and L. Milstein, "Error statistics in data transmission over fading channels," IEEE Trans. Commun., vol. 46, pp. 1468-1477, Nov. 1998. 